Pacific Journal of Mathematic 


\title{
THE BEHAVIOR OF SOLUTIONS OF ORDINARY, SELF-ADJOINT DIFFERENTIAL EQUATIONS OF ARBITRARY EVEN ORDER
}

\author{
ROBERT W. Hunt
}

Introduction. It is the purpose of this paper to establish some properties of the zeros of solutions of ordinary, self-adjoint differential equations of arbitrary even order of the form

$$
\left[r(x) y^{(n)}\right]^{(n)}+(-1)^{n+1} p(x) y=0
$$

where $r(x)>0, p(x)>0$, and both coefficients are continuous on $[a, \infty)$. Of particular concern is the existence of a nontrivial solution of (1) which satisfies one of the following sets of two-point boundary conditions

$$
\begin{aligned}
& y(a)=y^{\prime}(a)=\cdots=y^{(n-1)}(a)=0=y(b)=y^{\prime}(b)=\cdots=y^{(n-1)}(b) \\
& y(a)=y^{\prime}(a)=\cdots=y^{(n-1)}(a)=0=y_{1}(b)=y_{1}^{\prime}(b)=\cdots=y_{1}^{(n-1)}(b)
\end{aligned}
$$

where $y_{1}(x) \equiv r(x) y^{(n)}(x)$, a notation which will be continued throughout the discussion, and $b>a$.

Recently the special fourth-order case $(n=2)$ has been investigated extensively by W. Leighton and Z. Nehari [10], by H. M. and R. L. Sternberg [13], by H. C. Howard [8], and by J. H. Barrett [2, 3, 4]. In the present paper some of the methods of Barrett $[2,4]$ are extended to the general case; and, in so doing, some of the arguments used for $n=2$ are simplified.

W. T. Reid has recently announced [12] a general discussion including the above types of zeros of solutions of quasi-differential equations of even order of which (1) is a special case. Reid discusses related eigenvalue inequalities and his methods are variational in nature and assume some basic results of the spectral theory for boundary problems that have been established earlier in the study of the calculus of variations.

This discussion, which generalizes Barrett's methods, has the advantage that only fairly well-known properties of matrices and differential equations are used. Furthermore, and most important, a considerably stronger criterion for the existence of a non-trivial solution satisfying (2) (see Theorem 4.3) and of one satisfying (3) (see Corollary 5.1) is established by utilizing the simple form of (1). Then two comparison theorems, established by an application of Reid's variational results [12], extend these stronger results to the general self-adjoint

Received May 29, 1961. This paper is part of a thesis submitted to the faculty of the University of Utah in partial fulfillment of the requirements for the degree of Doctor of Philosophy. The author expresses thanks to the thesis director, Professor John H. Barrett. Presented to the American Mathematical Society, April 22, 1961. 
case, i.e., the differential equation of the form

$$
\left[r_{n}(x) y^{(n)}\right]^{(n)}+\sum_{i=0}^{n-1}\left[(-1)^{n+i+1} r_{i}(x) y^{(i)}\right]^{(i)}=0,
$$

where $r_{i}(x)>0$ for $i=0,1,2, \cdots, n$, and all of the coefficients are continuous on $[a, \infty)$. This extension is discussed in $\S 6$.

For the sake of completeness, there is developed in the first section a canonical representation of (1) as a system of two first-order matrix equations as given by H. Kaufman and R. L. Sternberg [9], as modified by Barrett [4], and as modified here by a method suggested by a problem in [6, problem 19, p. 206]. This system is of the form

$$
Y^{\prime}=E(x) Z, \quad Z^{\prime}=-F(x) Y,
$$

and is so designed that a singularity of the $n$ th-order matrix $Y(x)$ at $x=b$ gives the existence of a nontrivial solution of (1) satisfying the conditions (2); and a singularity of $Z(x)$ at $x=b$ gives the existence of a nontrivial solution of (1) satisfying the conditions (3). These properties are discussed in $\S 2$. In $\S 3$, the determinants of $Y(x)$ and $Z(x)$ are shown to satisfy certain second-order, self-adjoint differential equations which generalizes a result for the case $n=2$ due to Leighton and Nehari [10] and Barrett [2]. In $\S \S 4$ and 5, conditions for the existence of nontrivial solutions satisfying the conditions (2) and (3) are discussed.

1. A matrix differential system related to (1). This discussion parallels [4] with a slightly different modification suggested by a problem in [6, page 206]. Let $y(x)$ be any solution of (1) and let

$$
y_{1}(x) \equiv r(x) y^{(n)}(x),
$$

a notation which will be used throughout the discussion. Define the $n$ th-order column vectors $\alpha(x)$ and $\widetilde{\alpha}(x)$ by

$$
\alpha(x)=\left(\alpha_{i}\right)=\left(y^{(i-1)}\right), \widetilde{\alpha}(x)=\left(\widetilde{\alpha}_{i}\right)=\left((-1)^{i} y_{1}^{(n-i)}\right),
$$

where $i=1,2, \cdots, n$. Then $\alpha(x)$ and $\widetilde{\alpha}(x)$ satisfy the system

$$
\begin{aligned}
\alpha^{\prime} & =B \alpha+C(x) \widetilde{\alpha} \\
\tilde{\alpha}^{\prime} & =A(x) \alpha-B^{*} \widetilde{\alpha},
\end{aligned}
$$

where $A(x), B$, and $C(x)$ are $n \times n$ matrices defined as follows, ( $i$ denotes the row index and $j$ the column index).

where

$$
A(x)=\left(a_{i j}(x)\right) \text {, }
$$

$$
\begin{gathered}
a_{11}(x)=(-1)^{n+1} p(x), a_{i j}(x) \equiv 0 \text { for } i \neq 1 \text { or } j \neq 1 \\
B=\left(b_{i j}\right) \text {, where } b_{i j}=1 \text { for } j=i+1, b_{i j}=0 \text { for } j \neq i+1 . \\
C(x)=\left(c_{i j}(x)\right),
\end{gathered}
$$

where 


$$
c_{n n}(x)=(-1)^{n} / r(x), c_{i j}(x) \equiv 0 \text { for } i \neq n \text { or } j \neq n .
$$

Next, consider the $n \times n$ matrices $D(x)$ and $\widetilde{D}(x)$ which satisfy

$$
D^{\prime}=B D, D(a)=I_{n} \text { and } \widetilde{D}^{\prime}=-B^{*} \widetilde{D}, \widetilde{D}(a)=I_{n},
$$

respectively, where $I_{n}$ is the $n \times n$ identity matrix. For simplicity in the remainder of the paper, and with no loss in generality, let $a=0$. Now, the equations for $D(x)$ and $\widetilde{D}(x)$ can be solved to give

$$
\begin{aligned}
D(x)= & \left(d_{i j}(x)\right), \text { where } d_{i j}(x) \equiv 0 \text { for } i>j \text { and } \\
& d_{i j}(x)=x^{j-i} /(j-i) ! \text { for } i \leqq j . \\
\widetilde{D}(x)= & \left(\widetilde{d}_{i j}(x)\right), \text { where } \widetilde{d}_{i j}(x) \equiv 0 \text { for } i<j \text { and } \\
& \widetilde{d}_{i j}(x)=(-1)^{i-j} x^{i-j} /(i-j) \text { ! for } j \leqq i .
\end{aligned}
$$

Now, let $\beta(x)$ and $\widetilde{\beta}(x)$ be $n$ th-order vectors defied by the relations

$$
\alpha(x)=D(x) \beta(x), \widetilde{\alpha}(x)=(-1)^{n} \widetilde{D}(x) \widetilde{\beta}(x) .
$$

Substitution into (5) then yields

$$
\begin{aligned}
& \beta^{\prime}=E(x) \widetilde{\beta} \\
& \widetilde{\beta}^{\prime}=-F(x) \beta,
\end{aligned}
$$

where $E(x)$ and $F(x)$ are $n \times n$ matrices defined as follows.

$$
\begin{aligned}
E(x)= & \left(e_{i j}(x)\right), \text { where } \\
& e_{i j}(x)=(-1)^{i+j} x^{2 n-i-j} /(n-i) !(n-j) ! r(x), \\
F(x)= & \left(f_{i j}(x)\right), \text { where } f_{i j}(x)=x^{i+j-2} p(x) /(i-1) !(j-1) ! \\
& \text { for } i \leqq j \text { and } f_{i j}(x) \equiv f_{i j}(x) .
\end{aligned}
$$

Thus, $E(x)$ and $F(x)$ are symmetric, positive semi-definite matrices. $D(x), E(x)$, and $F(x)$, are generalizations of matrices used by Barrett [4] for $n=2$.

Let $\left\{u_{i}(x)\right\}, i=1,2,3, \cdots, n$ be a set of solutions of (1) which satisfy the boundary conditions

$$
\begin{aligned}
& {\left[u_{i}(x)\right]^{(j-1)}=0 \text { for } x=0 \text { and } i, j=1,2, \cdots, n .} \\
& {\left[u_{i, 1}(x)\right]^{(j-1)}=\delta_{i j} \text { for } x=0 \text { and } i, j=1,2, \cdots, n} \\
& \quad \text { and } u_{i, 1}(x)=r(x) u_{i}^{(n)}(x) .
\end{aligned}
$$

Now, denote the $n$th order vector with components $u_{i}^{(j-1)}(x), j=1$, $2, \cdots, n$, by $\alpha_{u_{i}}(x)$ and the $n$th order vector with components $(-1)^{j} u_{i, 1}^{(n-j)}(x), j=1,2, \cdots, n$, by $\widetilde{\alpha}_{u_{i}}(x)$. Then define

(8) $\beta_{u_{i}}(x)=D^{-1}(x) \alpha_{u_{i}}(x), \widetilde{\beta}_{u_{i}}(x)=(-1)^{n} \widetilde{D}^{-1}(x) \widetilde{\alpha}_{u_{i}}(x), i=1,2, \cdots, n$.

TheOREM 1.1. Let $Y(x)$ be an $n \times n$ matrix whose columns (in the 
order $i=1,2, \cdots, n)$ are the $\beta_{u_{i}}(x)$ and let $Z(x)$ be an $n \times n$ matrix whose columns are (in the order $i=1,2, \cdots, n$ ) the $\widetilde{\beta}_{u_{i}}(x)$. Then $Y(x)$ and $Z(x)$ satisfy

$$
Y^{\prime}=E Z, Z^{\prime}=-F Y
$$

Proof. This theorem can be verified by direct substitution.

2. Relations between the system (9) and equation (1). First, two types of zeros of solutions of (1) are defined as follows.

Definition 2.1. The number $\eta_{1}(0)$ is the smallest number $b$ on $(0, \infty)$ such that the boundary conditions

$$
y(0)=y^{\prime}(0)=\cdots=y^{(n-1)}(0)=0=y(b)=y^{\prime}(b)=\cdots=y^{(n-1)}(b)
$$

are satisfied nontrivially by a solution $y(x)$ of (1). This is the type of boundary problem considered in $[2,4,12,13]$.

DEFINITION 2.2. The number $\mu_{1}(0)$ is the smallest number $b$ on $(0, \infty)$ such that the boundary conditions

$$
y(0)=y^{\prime}(0)=\cdots=y^{(n-1)}(0)=0=y_{1}(b)=y_{1}^{\prime}(b)=\cdots=y_{1}^{(n-1)}(b)
$$

are satisfied nontrivially by a solution $y(x)$ of (1). This is a generalization of the type of condition first used by Barrett [2,4] and Howard [8] as an intermediate condition to (10).

THeOREM 2.1. A number $b \varepsilon(0, \infty)$ is the smallest number on that interval for which det $Y(b)=0$ if and only if $b=\eta_{1}(0)$ for (1). A number $b \varepsilon(0, \infty)$ is the smallest number on that interval for which det $Z(b)=0$ if and only if $b=\mu_{1}(0)$ for (1).

Proof. Note first of all that det $Y=W\left[u_{1}, \cdots, u_{n}\right]$ and $\operatorname{det} Z(x)$ $=W\left[u_{1,1}, u_{2,1}, \cdots, u_{n, 1}\right]$, where $W$ turns out to be a Wronskian in each case. The former is true since

$$
Y(x)=D^{-1}(x) \mathscr{f}(x),
$$

$\mathscr{F}(x)$ being the matrix of $W\left[u_{1}, u_{2}, \cdots, u_{n}\right]$, and $\operatorname{det} D(x) \equiv 1$. To verify that $\operatorname{det} Z(x)=W\left[u_{1,1}, u_{2,1}, \cdots, u_{n, 1}\right]$, observe that

$$
Z(x)=(-1)^{n} \widetilde{D}^{-1}(x) \xi(x),
$$

$\xi(x)$ being the matrix whose columns are the $\widetilde{\alpha}_{u_{i}}(x)$ in the order $i=1$, $2, \cdots, n$. But $\operatorname{det}(-1)^{n} y(x)=W\left[u_{1,1}, u_{2,1}, \cdots, u_{n, 1}\right]$ and $\operatorname{det} \widetilde{D}(x) \equiv 1$. Using the definition of the functions $u_{i}(x)$, the result now follows. 
2. Second-order, self-adjoint, linear differential equations which have det $Y(x)$ and det $Z(x)$ as solutions. To simplify the notation, let

$$
\operatorname{det} Y(x)=\sigma(x), \quad \operatorname{det} Z(x)=\rho(x)
$$

in the remainder of the discussion. Note that $\sigma(0)=0$ and $\rho(0)=1$. Consider, first, the following lemmas.

LEMma 3.1. Let $\tilde{\sigma}(x)$ denote the Wronskian, $W\left[u_{1}, u_{2}, \cdots, u_{n-1}\right]$ $(n>1)$ where the functions $u_{i}(x)$ are as defined in $\S 1$ and (7). Then $\widetilde{\sigma}(x) \neq 0$ on $(0, \infty)$.

Proof. Assume that $\widetilde{\sigma}(x)$ has a zero on $(0, \infty)$. Then there is a solution $y(x)$ of (1) which is a linear combination of $u_{1}(x), u_{2}(x), \cdots$, $u_{n-1}(x)$ and a minimum value $x=b$ on $(0, \infty)$ such that $y(0)=y^{\prime}(0)=$ $\cdots=y^{(n-1)}(0)=y_{1}^{(n-1)}(0)=0$ and $y(b)=y^{\prime}(b)=\cdots=y^{(n-2)}(b)=0$.

Let $y(x)$ have $m$ distinct zeros on $(0, b)(m \geqq 0)$, the first such zero being $x=c$ on $(0, b]$; and suppose, without loss of generality, that $y(x)>0$ on $(0, c)$. Then, because of the conditions on $y(x)$ at $x=0$, $y_{1}(0) \geqq 0$. Now, noting the condition on $y(x)$ at $x=0$ and $x=b$, Rolle's theorem can be applied to $y(x), y^{\prime}(x), \cdots, y_{1}^{(n-2)}(x)$ in turn to give the following information.

$y^{\prime}(x)$ has at least $m+1$ distinct zeros on $(0, b)$.

$y^{\prime \prime}(x)$ has at least $m+2$ distinct zeros on $(0, b)$.

- .

$y^{(n-1)}(x)$ has at least $m+n-1$ distinct zeros on $(0, b)$.

$y_{1}(x)$ has at least $m+n-1$ distinct zeros on $(0, b)$.

$y_{1}^{\prime}(x)$ has at least $m+n-2$ distinct zeros on $(0, b)$, the first one of which can be chosen to the right of the first of the above $m+n-1$ distinct zeros of $y_{1}(x)$.

$y_{1}^{\prime \prime}(x)$ has at least $m+n-3$ distinct zeros on $(0, b)$, the first one of which can be chosen to the right of the first of the above $m+n-2$ distinct zero of $y_{1}^{\prime}(x)$.

$y_{1}^{(n-1)}(x)$ has at least $m$ distinct zeros on $(0, b)$ if $m \neq 0$, the first one of which can be chosen to the right of the first of the above $m+1$ distinct zeros of $y_{1}^{(n-2)}(x)$.

(If $m=0$, the last statement is replaced by the statement that $y_{1}^{(n-1}(x) \neq 0$ on $(0, b)$.)

Then, from the last statement for $m \neq 0, y_{1}^{(n)}(x)$, and hence $y(x)$, has at least $m$ distinct zeros on $(0, b)$ since $y_{1}^{(n-1)}(0)=0$. If $m=0$, $y(x)$ has no zeros on $(0, b)$. But $y(x)$ has exactly $m$ distinct zeros on $(0, b)$. Thus, each of the functions $y_{1}^{(n-1)}(x), \cdots, y_{1}^{\prime \prime}(x), y_{1}^{\prime}(x)$ must have exactly the number of distinct zeros on $(0, b)$ as given in the preceding statements since each has at least that number of zeros and if any 
one had more distinct zeros, it would follow that $y(x)$ had more than $m$ distinct zeros. Also, all of the distinct zeros of $y(x)$ are simple.

Next, $y_{1}^{(n-1)}(x)=(-1)^{n} \int_{0}^{x} p(t) y(t) d t$. Consider the case where $n$ is even. Then $y_{1}^{(n-1)}(x)>0$ for $x$ on $(0, c)$. Thus, $y_{1}^{(n-2)}(x)$ must begin at $x=0$ with zero slope, have positive slope on $(0, c)$, and have a zero before its slope has a zero (since for $m \neq 0$ the first zero of $y_{1}^{(n-1)}(x)$ is to the right of the first zero of $y_{1}^{(n-2)}(x)$ on $(0, \infty)$ and for $m=0, y_{1}^{(n-1)}(x) \neq 0$ on $(0, b)$ by the above considerations). This is possible only if $y_{1}^{(n-2)}(0)$, $<0$. Then $y_{1}^{(n-3)}(x)$ must begin at $x=0$ with negative slope and have a zero before its slope has a zero which implies that $y_{1}^{(n-3)}(0)>0$. Iteration of this argument gives $y_{1}(0)<0$, a contradiction. For the case in which $n$ is odd, this contradiction is obtained similarly. Thus, $\tilde{\sigma}(x) \neq 0$ on $(0, \infty)$.

LEMma 3.2. Let $\tilde{\rho}(x)$ denote the Wronskian, $W\left[u_{1,1}, u_{2,1}, \cdots, u_{n-1,1}\right]$ $(n>1)$ where $u_{j, 1}(x)=r(x) u_{j}^{(n)}(x)$ and the functions $u_{i}(x)$ are as in Lemma 3.1. Then $\tilde{\rho}(x)>0$ on $[0, \infty)$.

Proof. This result is proved in the same way as the preceding lemma. $\tilde{\rho}(x)>0$ on $[0, \infty)$ since $\tilde{\rho}(0)=1$.

LEMma 3.3. The solutions $u_{i}(x)$, as previously defined, satisfy the following set of $n(n-1) / 2$ identities $(n>1)$ :

$$
\begin{aligned}
& \sum_{m=0}^{n-1}\left[(-1)^{m}\left(u_{i}^{(m)}(x) u_{i+j, 1}^{(n-m-1)}(x)-u_{i+j}^{(m)}(x) u_{i, 1}^{(n-m-1)}(x)\right)\right] \equiv 0, \\
& i=1,2, \cdots, n-1 \text { and } j=1,2, \cdots, n-i .
\end{aligned}
$$

Proof. The lemma is established by an induction on the following general observations:

$$
\left(r u_{i}^{(n)}\right)^{(n)}+(-1)^{n+1} p u_{i} \equiv 0, \quad\left(r u_{i+j}^{(n)}\right)^{(n)}+(-1)^{n+1} p u_{i+j} \equiv 0
$$

and hence $u_{i}\left(r u_{i+j}^{(n)}\right)^{(n)}-u_{i+j}\left(r u_{i}^{(n)}\right)^{(n)} \equiv 0$; that is, $u_{i} u_{i+j, 1}^{(n)}-u_{i+j} u_{i, 1}^{(n)} \equiv 0$. Then $n$ integrations by parts from 0 to $x$ give

$$
\begin{aligned}
\sum_{m=0}^{n-1}(-1)^{m} u_{i}^{(m)} u_{i+j, 1}^{(n-m-1)} & +(-1)^{n} \int_{0}^{x} u_{i}^{(n)} u_{i+j, 1} d t \\
& +\sum_{m=0}^{n-1}(-1)^{m+1} u_{i+j}^{(m)} u_{i, 1}^{(n-m-1)}+(-1)^{n+1} \int_{0}^{x} u_{i+j}^{(n)} u_{i, 1} \equiv 0,
\end{aligned}
$$

from which the lemma follows.

Next, note that the function $\left(r(x) \sigma^{\prime}(x)\right)^{\prime}$ is as follows:

$$
\left(r(x) \sigma^{\prime}(x)\right)^{\prime}=D_{1}(x)+D_{2}(x),
$$

where $D_{1}(x)$ is an $n$th order determinant whose first $n-1$ rows are 
$\left(u_{1}^{(j)} u_{2}^{(j)} \cdots u_{n}^{(j)}\right), j=0, \cdots, n-3, n-1$, and whose last row is $\left(u_{1,1}, u_{2,1}\right.$ $\left.\cdots u_{n, 1}\right)$; and $D_{2}(x)$ is an $n$th order determinant whose first $n-1$ rows are the same as those of $D_{1}(x)$ except that $j=0, \cdots, n-2$, and whose last row is $\left(u_{1,1}^{\prime}, u_{2,1}^{\prime} \cdots u_{n, 1}^{\prime}\right)$.

Lemma 3.4. $\left(r(x) \sigma^{\prime}(x)\right)^{\prime}$ can be written as

$$
\left(r(x) \sigma^{\prime}(x)\right)^{\prime} \equiv 2 D_{1}(x)
$$

In other words, the two determinants on the right-hand side of (13) are identically equal.

Proof. Let $W_{i, i+j}$ denote the sub-Wronskian of $W\left[u_{1}, u_{2}, \cdots, u_{n}\right]$ which is obtained from the latter by deletion of the $i$ th and $(i+j)$ th columns and the last two rows. Then, using the equations (12), consider

$$
\sum_{m=0}^{n-1}\left[(-1)^{m}(-1)^{2 i+j+1} W_{i, i+j}\left(u_{i}^{(m)} u_{i+j, 1}^{(n-m-1)}-u_{i+j}^{(m)} u_{i, 1}^{(n-m-1)}\right] \equiv 0,\right.
$$

for $i=1,2, \cdots, n-1$ and $j=1,2, \cdots, n-i$. Summing these $n(n-1) / 2$ identities yields

$$
\sum_{i=1}^{n-1} \sum_{j=1}^{n-i} \sum_{m=0}^{n-1}\left[(-1)^{m}(-1)^{2 i+j+1} W_{i, i+j}\left(u_{i}^{(m)} u_{i+j, 1}^{(n-m-1)}-u_{\imath+j}^{(m)} u_{i, 1}^{(n-m-1)}\right)\right] \equiv 0
$$

Now take $m=n-1$. This gives

$$
\sum_{i=1}^{n-1} \sum_{j=1}^{n-i}\left[(-1)^{n-1}(-1)^{2 i+j+1} W_{i, i+j}\left(u_{i}^{(n-1)} u_{i+j, 1}-u_{i+j}^{(n-1)} u_{i, 1}\right)\right]
$$

which is exactly the first determinant on the right-hand side of (13) if $n$ is odd and the negative of the determinant if $n$ is even. This can be seen by expanding the determinant by Laplace's development based upon minors of the last two rows.

Next take $m=n-2$. Then, as above, this gives the negative of the second determinant on the right side of (13) if $n$ is odd and the determinant if $n$ is even.

The result (13') will now follow if it can be shown that

$$
\sum_{i=1}^{n-1} \sum_{j=1}^{n-i} \sum_{m=0}^{n-3}\left[(-1)^{m}(-1)^{2 i+j+1} W_{i, i+j}\left(u_{i}^{(m)} u_{i+j, 1}^{(n-m-1)}-u_{i+j}^{(m)} u_{i, 1}^{(n-m-1)}\right)\right] \equiv 0 .
$$

In particular, this holds for each $m$ fixed between 0 and $n-3$ as follows. Fix $m$ so that $0 \leqq m \leqq n-3$. Then consider the identically zero determinant (two identical rows) 


$$
\begin{aligned}
& \left|\begin{array}{llll}
u_{1} & u_{2} & \cdots & u_{n} \\
u_{1}^{\prime} & u_{2}^{\prime} & \cdots & u_{n}^{\prime} \\
\cdot & \cdot & \cdots & \\
u_{1}^{(n-3)} & u_{2}^{(n-3)} & \cdots & u_{n}^{(n-3)} \\
u_{1}^{(m)} & u_{2}^{(m)} & \cdots & u_{n}^{(m)} \\
u_{1,1}^{(n-m-1)} & u_{2,1}^{(n-m-1)} & \cdots & u_{n, 1}^{(n-m-1)}
\end{array}\right| \\
& \equiv \sum_{i=1}^{n-1} \sum_{j=1}^{n-i}\left[(-1)^{2 i+j+2 n-1} W_{i, i+j}\left(u_{i}^{(m)} u_{i+j, 1}^{(n-m-1)}-u_{i+j}^{(m)} u_{i, 1}^{(n-m-1)}\right)\right],
\end{aligned}
$$

the expansion being by Laplace's development based upon minors of the last two rows. But this expansion is either equal to the above with a fixed $m$ or the negative of the above with a fixed $m$. Thus, the assertion holds; and (13') follows.

THEOREM 3.1. The function $\sigma(x)=\operatorname{det} Y(x)$ is a solution of

$$
\left(r \sigma^{\prime} / \tilde{\sigma}^{2}\right)^{\prime}+2 r\left(\delta / \tilde{\sigma}^{3}\right) \sigma=0,
$$

on $(0, \infty)$, where $\delta(x)$ is a determinant of order $n-1$ whose rows are $\left(u_{1}^{(j)} u_{2}^{(j)} \cdots n_{n-1}^{(j)}\right)$, where $j=0, \cdots, n-3, n(n>1)$.

Proof. Using $\left(13^{\prime}\right)$, the differential equation (14) can be written in the form $(2 / \widetilde{\sigma})\left(D_{1} \widetilde{\sigma}-r \tilde{\sigma}^{\prime} \sigma^{\prime}+r \delta \sigma\right)=0$. Now, if it can be established that the left hand side of this equation is identically zero on $(0, \infty),(14)$ will be established. To show this, consider the following identically zero determinant (can be verified by the use of induction and expansion by minors) and its Laplace development based upon minors of the first $n-1$ columns.

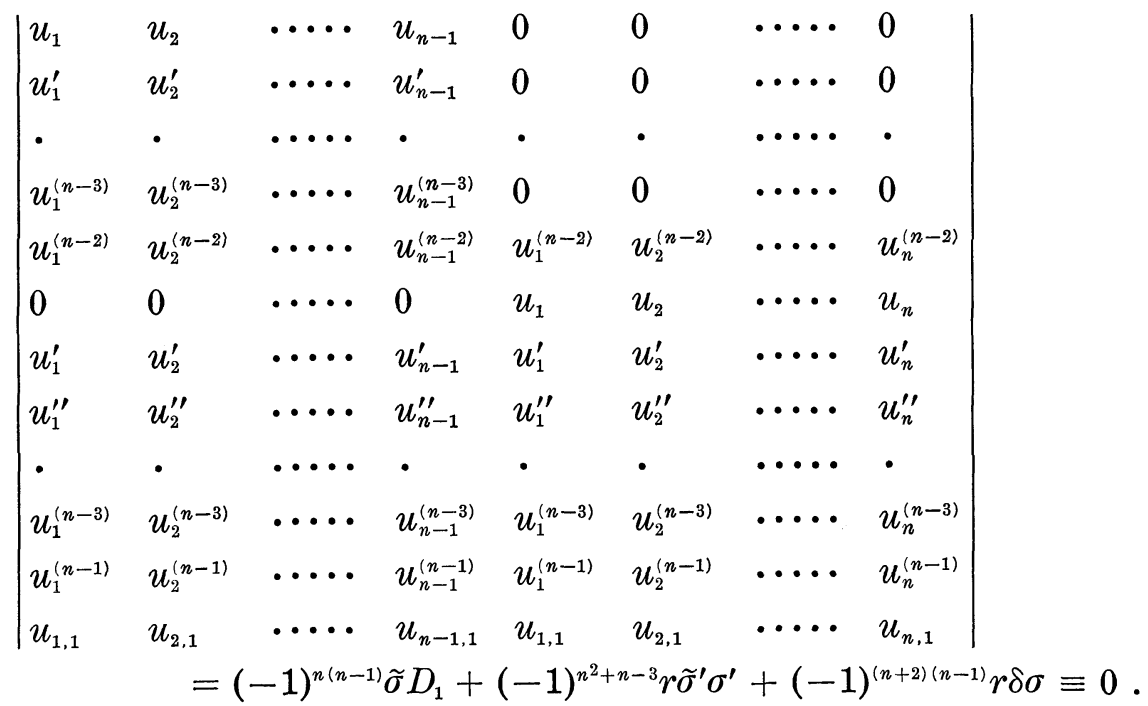

The right hand side of this equation is exactly the term which was to 
be shown identically zero. This completes the proof of the theorem.

An analogous result to Theorem 3.1 for $\rho(x)=\operatorname{det} Z(x)$ is given as follows.

THEOREM 3.2. The function $\rho(x)=\operatorname{det} Z(x)$ is a solution of

$$
\left(\rho^{\prime} \mid p \tilde{\rho}^{2}\right)^{\prime}+(2 / p)\left(\delta_{1} / \widetilde{\rho}^{3}\right) \rho=0,
$$

on $(0, \infty)$, where

$$
\delta_{1}(x)=\left|\begin{array}{llll}
u_{1,1} & u_{2,1} & \cdots & u_{n-1,1} \\
u_{1,1}^{\prime} & u_{2,1}^{\prime} & \cdots & u_{n-1,1}^{\prime} \\
\cdot & \cdot & \cdots & \cdot \\
u_{1,1}^{(n-3)} & u_{2,1}^{(n-3)} & \cdots & u_{n-1,1}^{(n-3)} \\
u_{1,1}^{(n)} & u_{2,1}^{(n)} & \cdots & \begin{array}{c}
(n) \\
n-1,1
\end{array}
\end{array}\right|(n>1) .
$$

4. Conditions for the existence of $\mu_{1}(0)$. This first two theorems of this section generalize results for the case $n=2$ by Barrett in [2, 4]. These results could, also, be obtained by specialization of Reid's work in [12]. Theorem 4.3, on the other hand, generalizes Barrett's Lemma 2.6 in [2], gives an improvement over Theorem 4.2, and gives a condition for the existence of $\mu_{1}(0)$ which improves the specialization obtained from [12].

THEOREM 4.1. If $\eta_{1}(0)$ exists, then $\mu_{1}(0)$ exists and $0<\mu_{1}(0) \leqq \eta_{1}(0)$.

Proof. $\operatorname{det} Z(0)=1$ and thus there is a maximum value $x=b$ on $(0, \infty]$ such that $Z(x)$ is nonsingular on $[0, b)$. On $[0, b)$, lat $K(x)=$ $Y(x) Z^{-1}(x)$ so that $K(x)$ is symmetric and

$$
K^{\prime}(x)=E(x)+K(x) F(x) K(x), K(0)=0 .
$$

$K(x)$ has been discussed and utilized in [1] and [11], as well as [4] where Barrett has used it for the case $n=2$. Now let $\xi=\left(\xi_{i}\right)$ be an $n$th order, constant, column vector. Then, using (18),

$$
\begin{aligned}
& \xi^{*} K(x) \xi \geqq \int_{0}^{x} \xi^{*} E(t) \xi d t \\
& =\int_{0}^{x} \frac{1}{r(t)}\left[\frac{\xi_{1} t^{n-1}}{(n-1)}-\xi_{2} t^{n-2}+\xi_{3}(n-2) t^{n-3}-\cdots+(-1)^{n-1} \xi_{n}(n-2) !\right]^{2} d t,
\end{aligned}
$$

and hence $K(x)$ is positive definite on $(0, b)$. Thus, $\operatorname{det} K(x)=$ det $Y(x) / \operatorname{det} Z(x)$ is positive and $Y(x)$ is nonsingular on $(0, b)$. This implies that $\eta_{1}(0)$ does not exist on $(0, b)$ and the result of the theorem follows. 
THEOREM 4.2. If $\int^{\infty} x^{2(n-1)} p(x) d x=\infty$, then $\mu_{1}(0)$ exists.

Proof. Suppose that $\int^{\infty} x^{2(n-1)} p(x) d x=\infty$ and $\operatorname{det} Z(x)>0$ on $[0, \infty)$. Then by the preceding theorem, det $Y(x)>0$ on $(0, \infty)$ and the matrix $K(x)=Y(x) Z^{-1}(x)$ is positive definite on $(0, \infty)$. Thus, for every constant vector $\xi=\left(\xi_{i}\right),(i=1,2, \cdots, n)$, it follows that

$$
\xi^{*} K^{-1} K^{\prime} K^{-1} \xi \geqq \xi^{*} F \xi=p(x)\left[\xi_{1}+\sum_{i=2}^{n} \xi_{i} x^{i-1} /(i-1)\right]^{2}
$$

Hence, if $x_{0} \in(0, \infty)$ and $x \in\left(x_{0}, \infty\right)$, it follows that

$$
\begin{aligned}
\int_{x 0}^{x} p(t) & {\left[\xi_{1}+\sum_{i=2}^{n} \xi_{i} t^{i-1} /(i-1)\right]^{2} d t } \\
& =\int_{x_{0}}^{x} \xi^{*} F(t) \xi d t<\xi^{*} K^{-1}\left(x_{0}\right) \xi<\infty,
\end{aligned}
$$

and the theorem follows.

In order to use to advantage the second-order equation (16) which $\rho(x)$ satisfies (as Barrett does in [2]), it must first be established that the coefficients in (16) are positive on $(0, \infty)$. Lemma 3.2 gives $\widetilde{\rho}(x)>0$ on $[0, \infty)$. Thus, it remains to be shown that $\delta_{1}(x)$, as defined by (17), is positive on $(0, \infty)$. In fact, it is necessary to know that a certain family of determinants (which includes $\delta_{1}(x)$ ) contains only determinants which are nonzero on $(0, \infty)$ and that certain of these (including $\delta_{1}(x)$ ) are positive on $(0, \infty)$. These results are given in Lemma 4.1.

Consider the matrix $M(x)$ with $2 n$ rows and $n-1$ columns $(n>1)$ whose first $n$ rows are, in order, the row vectors $\left(u_{1}^{(k)} u_{2}^{(k)} \cdots u_{n-1}^{(k)}\right), k=$ $0,1, \cdots, n-1$, and whose last $n$ rows are, in order, the row vectors $\left(u_{1,1}^{(k)} u_{2,1}^{(k)} \cdots u_{n-1,1}^{(i)}\right), k=0,1, \cdots, n-1$. Let $\mathscr{F}$ denote the family of determinants of order $n-1$ obtained from $M(x)$ by deleting $n+1$ rows and taking the determinant of the resulting square matrix. Note that for every determinant $D(x)$ in $\mathscr{F}, D(0)=0$, except the case $D(x)=$ $\tilde{\rho}(x)$ when $D(0)=1$. Two subsets of $\mathscr{F}$ are the sets $\left\{\delta_{j}(x) / p(x)\right\}$ and $\left\{\gamma_{j}(x)\right\}, j=1, \cdots, n-2(n>2)$ defined as follows:

DEFINITION 4.1. $\delta_{j}(x) / p(x)$ is a determinant of order $n-1$ with first row $\left(u_{1}, u_{2} \cdots u_{n-1}\right)$ and remaining rows, in order, $\left(u_{1,1}^{(k)} u_{2,1}^{(k)} \cdots u_{n-1,1}^{(k)}\right), k=$ $0,1, \cdots, n-j-2, n-j, \cdots, n-2$.

DEFINITION 4.2. $\gamma_{j}(x)$ is a determinant of order $n-1$ with rows, in order, $\left(u_{1,1}^{(k)} u_{2,1}^{(k)} \cdots u_{n-1,1}^{(k)}\right), k=0,1, \cdots, n-j-3, n-j-1, \cdots, n-1$.

Lemma 4.1. If $D(x)$ is any determinant belonging to $\mathscr{F}$, then $D(x) \neq 0$ on $(0, \infty)$. Furthermore, if $\widetilde{D}(x)$ is any determinant in the 
set $\left\{\delta_{j}(x) / p(x)\right\}$ or in the set $\left\{\gamma_{j}(x)\right\}, j=1, \cdots, n-2$, then $\widetilde{D}(x)>0$ on $(0, \infty)$.

Proof. A general determinant $D(x)$ in $\mathscr{F}$ will have the form:

$$
D(x)=\left|\begin{array}{llll}
u_{1,1}^{\left(i_{1}\right)} & u_{2}^{\left(i_{1}\right)} & \cdots & u_{n-1}^{\left(i_{1}\right)} \\
u_{1}^{\left(i_{2}\right)} & u_{2}^{\left(i_{2}\right)} & \cdots & u_{n-1}^{\left(i_{2}\right)} \\
\cdot & \cdot & \cdots & \cdot \\
u_{1}^{\left(i_{k}\right)} & u_{2}^{\left(i_{k}\right)} & \cdots & u_{n-1}^{\left(i_{k}\right)} \\
u_{1,1}^{\left(i_{k+1}\right)} & u_{2,1}^{\left(i_{k+1}\right)} & \ldots & u_{n-1,1}^{\left(i_{k+1}\right.} \\
\cdot & \cdot & \ldots & \cdot \\
u_{1,1}^{\left(i_{n-1}\right)} & u_{2}^{\left(i_{n-1}\right)} & \ldots & u_{n-1,1}^{\left(i_{n-1}\right)}
\end{array}\right|,
$$

where for some $k, 0 \leqq k \leqq n-1,\left\{i_{j}\right\}_{j=1}^{j=k}$ are some $k$ of the integers between 0 and $n-1$ and $0 \leqq i_{1}<i_{2}<\cdots<i_{k} \leqq n-1$; and $\left\{i_{j}\right\}_{j=k+1}^{j=n-1}$ are some $n-1-k$ of the integers between 0 and $n-1$ and $0 \leqq i_{k+1}$ $<i_{k+2}<\cdots<i_{n-1} \leqq n-1$.

The method of proof of the first part of the lemma, which will now be outlined, is the same as that of Lemmas 3.1 and 3.2 except for the added generality. In fact these two lemmas are included in the present lemma, but were established separately and in the last section for clarity.

Assume that $D(x)$ has a zero on $(0, \infty)$. Then there is a solution $y(x)$ of (1) which is a linear combination of $u_{1}(x), u_{2}(x), \cdots, u_{n-1}(x)$ and a minimum value $x=b$ on $(0, \infty)$ such that $y(0)=y^{\prime}(0)=\cdots=y^{(n-1)}(0)$ $=y_{(1)}^{(n-1)}(0)=0 \quad$ and $\quad y^{\left(i_{1}\right)}(b)=y^{\left(i_{2}\right)}(b)=\cdots=y^{\left(i_{k}\right)}(b)=y_{1}^{\left(i_{k+1}\right)}(b)=\cdots=$ $y_{1}^{\left(i_{n-1}\right)}(b)=0$. Recall that $y_{1}(x) \equiv r(x) y^{(n)}(x)$. Rolle's theorem can now be applied successively to $y(x)$ and, as in Lemma 3.1, a contradiction to the assumption is obtained to give the first part of the lemma.

The technique for establishing the second part of the lemma involves an iterated differentiation procedure which will now be described. Since, for all $j, \delta_{j}(0) / p(0)=0$ and $\gamma_{j}(0)=0$, a straightforward method of establishing the desired result should be that of showing that for each $j$ the first nonvanishing derivative of $\delta_{j}(x) / p(x)$ at $x=0$ or of $\gamma_{j}(x)$ at $x=0$ is positive. This requires modification, however, since the assumptions on the continuity of $p(x)$ are not sufficient to obtain all of the necessary derivatives directly.

Note the determinant forms of $\delta_{j}(x) / p(x)$ and $\gamma_{j}(x)$. The following discussion applies equally well to members of either of the sets of determinants. Differentiate the determinant $\widetilde{D}(x)$ successively until either:

(i) One of the determinants arising in the differentiation process is a positive constant times $\tilde{\rho}(x)$, or 
(ii) One or more of the determinants arising in the differentiation process has the row vector $\left(u_{1,1}^{(n)}, u_{2,1}^{(n)} \cdots u_{n-1,1}^{(n)}\right)$ in the last row and some vector other than $\left(u_{1}, u_{2} \cdots u_{n-1}\right)$ in the first row (for in this case the determinant is zero since $\left.u_{j, 1}^{(n)}(x) \equiv(-1)^{n} p(x) u_{j}(x)\right)$.

If (i) occurs first or in the same step as (ii) after $m$ differentiations, then $\widetilde{D}^{(h)}(0)=0$ for $h=1, \cdots, m-1$ since all of the determinants obtained belong to $\mathscr{F}$ and $\widetilde{D}^{(m)}(0)=k \tilde{\rho}(0)=k$ for some positive constant $k, k$ being the number of times that determinant has appeared in the differentiation process. Thus, $\widetilde{D}(x)>0$ on $(0, \infty)$ since $\widetilde{D}(x) \neq 0$ on $(0, \infty)$ and its first nonvanishing derivative at $x=0$ exists and is positive.

If (ii) occurs first after $m$ differentiations, the determinants of the form given in (ii) can be altered in form by replacing the last row by $(-1)^{n} p(x)\left(u_{1}, u_{2} \cdots u_{n-1}\right)$ and then bringing this row to the position of the first row and letting rows $1,2, \cdots, n-2$ become rows $2,3, \cdots$, $n-1$, respectively. The latter operation requires $n-2$ row interchanges so that each of these determinants is now some member of $\mathscr{F}$ (and not of the sets $\left\{\delta_{j} / p\right\}$ or $\left\{\gamma_{j}\right\}$ ) multiplied by $+p(x)$. Let $x_{0}$ be some positive finite number and denote the maximum and minimum values of $p(x)$ on $\left[0, x_{0}\right]$ by $p_{M}$ and $p_{m}$, respectively. Then, for each of these determinants multiplied by $p(x)$, replace $p(x)$ by $p_{m}$ if that determinant is positive on $(0, \infty)$ and replace $p(x)$ by $p_{M}$ if that determinant is negative on $(0, \infty)$. Recall that each determinant is nonzero by the first part of the lemma. On $\left[0, x_{0}\right]$, let $f_{1}(x)$ denote the new function obtained from $\widetilde{D}^{(m)}(x)$ by making these changes in certain determinants of $\widetilde{D}^{(m)}(x)$. Then $\widetilde{D}^{(m)}(x) \geqq f_{1}(x)$ on $\left[0, x_{0}\right]$ and $\widetilde{D}^{(m)}(0)=$ $f_{1}(0)=0$.

Next, on $\left[0, x_{0}\right]$, defferentiate $f_{1}(x)$ successively until (i) or (ii) occurs and then repeat the entire process just described. This process can be continued until for some minimum integer $h$ and some integer $q$, a function $f_{h}^{(q)}(x)$ (on $\left[0, x_{0}\right]$ ) with the following properties is obtained. $f_{h}^{(q)}(0)=k \tilde{\rho}(0)=k>0$, and $f_{h}^{(j)}(0)=0$ for $j=0,1, \cdots, q-1$. Then $f_{h}(x)>0$ in some right-hand neighborhood of $x=0$. Finally, from the set of inequalities obtained, from the fact that each $f_{j}(x)(j=1,2, \cdots, h$ if $q>0$ and $j=1,2, \cdots, h-1$ if $q=0$ ) and its appropriate derivatives are all zero for $x=0$, and from the first part of the lemma, it follows that $\widetilde{D}(x)>0$ on $(0, \infty)$.

The following generalization of a theorem of Barrett [2] gives a weaker condition for the existence of a $\mu_{1}(0)$ for (1) and thus a stronger result than Theorem 4.2 (and hence a better result than Reid's results [12] give for this case since Theorem 4.2 coincides with Reid's result in [12]).

THEOREM 4.3. If $\int^{\infty} p(x)\left({ }_{x_{0}}^{x_{n}} p\right)^{2} d x=\infty$, where $\stackrel{x}{I}^{n} p$ is the $n$th iter- 
ated integral of $p(x)$, then $\mu_{1}(0)$ exists.

Proof. From Lemma 3.2 and Lemma 4.1, it follows that (16) has positive coefficients on $(0, \infty)$. Since $\rho(0)=1, \rho^{\prime}(0)=0$, a well-known result for second-order, self-adjoint differential equations [2, Theorem 1.3] can be applied to give the existence of a zero of $\rho(x)$ on $(0, \infty)$ if it is known that $\int^{\infty} p(x) \widetilde{\rho}^{2}(x) d x=\infty$. But, using Theorem 2.1, a zero of $\rho(x)$ on $(0, \infty)$ implies the existence of a $\mu_{1}(0)$.

Thus, Theorem 4.3 will be proved if it can be shown that

$$
\tilde{\rho}(x) \geqq k{\underset{x}{x_{0}}}_{I_{n}}^{x_{n}} p, \quad x_{0}>0, k>0,
$$

for then the hypothesis of the theorem implies that the desired property, $\int^{\infty} p(x) \tilde{\rho}^{2}(x) d x=\infty$, is true.

Now, $\tilde{\rho}(x)=W\left[u_{1,1}(x), u_{2,1}(x), \cdots, u_{n-1,1}(x)\right]$ and differentiation yields $\tilde{\rho}^{\prime \prime}(x)=\gamma_{1}(x)+\delta_{1}(x)$, where $\gamma_{1}(x)$ and $\delta_{1}(x)$ are as previously defined. Then, consider

$$
\tilde{\rho}^{\prime \prime}(x)-\delta_{1}(x) \text { and }\left[\tilde{\rho}^{\prime \prime}(x)-\delta_{1}(x)\right]^{\prime}=\gamma_{2}(x)+\delta_{2}(x) \text {. }
$$

Next, $\left[\left(\tilde{\rho}^{\prime \prime}-\delta_{1}\right)^{\prime}-\delta_{2}\right]^{\prime}=\gamma_{3}+\delta_{3}$ is obtained, and the process can be continued to obtain finally

$$
\left[\left(\cdots\left(\left(\left(\tilde{\rho}^{\prime \prime}-\delta_{1}\right)^{\prime}-\delta_{2}\right)^{\prime}-\delta_{3}\right)^{\prime}-\cdots-\delta_{n-4}\right)^{\prime}-\delta_{n-3}\right]^{\prime}=\gamma_{n-2}+\delta_{n-2},
$$

where $\gamma_{j}(x)$ and $\delta_{j}(x), j=1, \cdots, n-2$, are as previously defined.

Then, one more differentiation gives

$$
\left[\left(\cdots\left(\left(\left(\tilde{\rho}^{\prime \prime}-\delta_{1}\right)^{\prime}-\delta_{2}\right)^{\prime}-\delta_{3}\right)^{\prime}-\cdots-\delta_{n-3}\right)^{\prime}-\delta_{n-2}\right]^{\prime}=p(x) \delta^{*}(x),
$$

where $\delta^{*}(x)$ is a determinant of order $n-1$ with first row $\left(u_{1}, u_{2} \ldots\right.$ $u_{n-1}$ ) and remaining rows

$$
\left(u_{1,1}^{(k)}, u_{2,1}^{(k)} \cdots u_{n-1,1}^{(k)}\right) \text { for } k=1,2, \cdots, n-2 .
$$

Now, by using Lemma 4.1 and the fact that $\gamma_{n-2}^{\prime}=p(x) \delta^{*}(x), \delta^{*}(x)$ must be positive on $(0, \infty)$. Also, by the techniques of Lemma 4.1, $\delta^{* \prime}(x)$ is positive on $(0, \infty)$. Thus $\delta^{*}(x)$ is an increasing function on $(0, \infty)$ and for any $x_{0}>0$,

$$
\int_{x_{0}}^{x} p(t) \delta^{*}(t) d t \geqq \delta^{*}\left(x_{0}\right) \int_{x_{0}}^{x} p(t) d t=k \underset{x_{0}}{\stackrel{x_{n}}{I} p, k>0 .}
$$

Now, using Lemma 4.1, the expression

$$
\left[\left(\cdots\left(\left(\left(\tilde{\rho}^{\prime \prime}-\delta_{1}\right)^{\prime}-\delta_{2}\right)^{\prime}-\delta_{3}\right)^{\prime}-\cdots-\delta_{n-3}\right)^{\prime}-\delta_{n-2}\right] \geqq k \underset{x_{0}}{I} p
$$


can be integrated $n-1$ times and the inequality preserved. This gives $\tilde{\rho}(x) \geqq k I_{x_{0}}^{x} p$, the result needed to complete the proof of the theorem.

Note that for $0<x_{0}<x_{1} \leqq x<\infty$,

$$
\stackrel{x}{I}_{x_{0}}^{n} p=\int_{x_{0}}^{x} \int_{x_{0}}^{t} \cdots \int_{x_{0}}^{u} p(v) d v \geqq \frac{\left(x-x_{1}\right)^{n-1}}{(n-1) !} \int_{x_{0}}^{x_{1}} p(x) d x,
$$

and thus Theorem 4.2 follows from Theorem 4.3. Hence, as remarked previously, Theorem 4.3 gives the better condition for the existence of $\mu_{1}(b)$ for any $b \varepsilon[0, \infty)$.

5. Conditions for the existence of $\eta_{1}(0)$. Consider the matrix $H(x)$ $=-Z(x) Y^{-1}(x)$ defined on $\left(0, \eta_{1}(0)\right)$. Note that $H(x)$ is then equal to $-K^{-1}(x)$, where $K(x)$ is the matrix used in Theorem 4.1 and satisfying (18). Then $H(x)$ is symmetric on $\left(0, \eta_{1}(0)\right)$ and satisfies

$$
H^{\prime}(x)=F(x)+H(x) E(x) H(x),
$$

as in [1] and [11].

Note that $H(x)$ is defined and symmetric on $(0, \infty)$ if there does not exist an $\eta_{1}(0)$. Then consider the following lemma.

LEMma 5.1. If $\eta_{1}(0)$ does not exist for (1), then all the eigenvalues of $H(x)$ are nondecreasing on $(0, \infty)$.

Proof. From the properties of the matrices $E(x)$ and $F(x)$ as discussed in $\S 1$ and from (19), it follows that $H^{\prime}(x) \geqq 0$ on $(0, \infty)$ if the inequality is used to imply positive semi-definiteness of $H^{\prime}(x)$. Then $H\left(x_{2}\right)-H\left(x_{1}\right)$ is positive semi-definite if $0<x_{1} \leqq x_{2}<\infty$, i.e., $H\left(x_{2}\right) \geqq$ $H\left(x_{1}\right)$. Then, from classical extremizing properties of eigenvalues, the result of the lemma follows.

In the present notation, $\mu_{1}(0)$ denotes the first zero of $\rho(x)$ on $(0, \infty)$. In addition, let $\mu_{2}(0)$ be the second distinct zero of $\rho(0)$ on $(0, \infty), \mu_{3}(0)$ the third distinct zero of $\rho(x)$ on $(0, \infty)$, and so forth.

TheOREM 5.1. If $\mu_{1}(0), \mu_{2}(0), \mu_{3}(0), \cdots, \mu_{n}(0), \mu_{n+1}(0)$ all exist (i.e., if $\rho(x)$ has $n+1$ distinct zeros on $(0, \infty))$, then $\eta_{1}(0)$ exists.

Proof. Suppose $\eta_{1}(0)$ does not exist so that Lemma 5.1 applies. Also, $H(x)=-Z(x) Y^{-1}(x)$ is defined on $(0, \infty)$ and has singularities at the $\mu_{i}(0)$ so that $\operatorname{det} H\left(\mu_{i}(0)\right)=0, i=1,2, \cdots, n+1$. But det $H(x)$ is equal to the product of the eigenvalues of $H(x)$ and thus, by Lemma 5.1 , det $H(x)$ can vanish at most $n$ times on $(0, \infty)$ unless det $H(x) \equiv 0$ on a subinterval of $(0, \infty)$. Assume the latter is true. Then $\operatorname{det} Z(x)$ $=\rho(x) \equiv 0$ on a subinterval of $(0, \infty)$. But $\rho(0)=1$ and $\rho(x)$ satisfies 
the linear, second order, self-adjoint differential equation (16) which implies that $\rho(x)$ cannot be identically zero on a sub-interval of $(0, \infty)$ without being identically zero on the entire half line. This contradiction completes the proof of the theorem.

THEOREM 5.2. If $\mu_{1}(0)$ exists and $\int^{\infty} d x / r(x)=\infty$, then $\eta_{1}(0)$ exists.

Proof. Assume that $\mu_{1}(0)$ does not exist. Then, using Lemma 5.1 and Theorem 5.1, it follows that the maximum eigenvalue of $H\left(\mu_{1}(0)\right)$ $=0$ and that there is a value $x=x_{0}$ on $\left(\mu_{1}(0), \infty\right)$ such that the maximum eigenvalue of $H(x)$ is positive on $\left[x_{0}, \infty\right)$. Furthermore, there is a value $x=x_{1} \geqq x_{0}$ such that $H(x)$ is nonsingular on $\left[x_{1}, \infty\right)$. Then, from (19), $H^{-1}(x) H^{\prime}(x) H^{-1}(x) \geqq E(x)$ on $\left[x_{1}, \infty\right)$. Also, if $\xi$ is any constant column vector of unit length, then on $\left[x_{1}, \infty\right)$,

$$
\begin{aligned}
\xi^{*} H^{-1}(x) \xi \leqq \max \text { eigenvalue of } & H^{-1}\left(x_{1}\right) \\
& -\min \text { eigenvalue } \int_{x_{1}}^{x} E(t) d t .
\end{aligned}
$$

Now, noting the form of $\xi^{*}\left(\int_{0}^{x} E(t) d t\right) \xi$ (as shown in the proof of Theorem 4.1), where $\xi$ is an arbitrary, nonzero, $n$ th-order, constant vector, $\int^{\infty} d x / r(x)=\infty$ implies that $\xi^{*}\left(\int_{0}^{\infty} E(t) d t\right) \xi=\infty$. But Reid [12] has proved a result which, in the terminology of the present problem, states that if $\xi^{*}\left(\int_{0}^{\infty} E(t) d t\right) \xi=\infty$ for arbitrary, nonzero, constant vectors, then the minimum eigenvalue of $\int_{0}^{x} E(t) d t$ approaches $\infty$ as $x$ approaches $\infty$. Then, it follows from (20) that there is a values $x=x_{2}$ on $\left(x_{1}, \infty\right)$ such that $H^{-1}(x)$ and $H(x)$ are negative definite on $\left[x_{2}, \infty\right)$. This contradicts the fact that the maximum eigenvalue of $H(x)$ is positive on $\left[x_{0}, \infty\right)$. This contradiction gives the result of the theorem.

Theorem 5.2 can be obtained from Reid's results in [12]. The proof given here is entirely different, however, from that in [12].

COROLLARY 5.1. If $\int^{\infty} p(x)\left(\underline{x}_{x_{0}}^{x_{n}} p\right)^{2} d x=\infty$ and $\int^{\infty} d x / r(x)=\infty$, then $\eta_{1}(0)$ exists $(n>1)$.

Proof. This result follows immediately by combining Theorems 4.3 and 5.2.

6. Conditions for the existence of $\mu_{1}(0)$ and $\eta_{1}(0)$ for (4). From the general results of Reid's [12], two theorems can be obtained and then used to prove two comparison theorems pertinent to the present dis- 
cussion. Then a comparison of (1) and (4) can be give conditions for the existence of $\mu_{1}(0)$ and $\eta_{1}(0)$ for (4), a more general self-adjoint equation then (1). For (4), $\eta_{1}(0)$ is defined as in Definition 2.1 and (10). However, for (4), the definition of $\mu_{1}(0)$ is obtained by altering Definition 2.2 by replacing (11) by

$$
y(0)=y^{\prime}(0)=\cdots=y^{(n-1)}(0)=0=y_{1}(b)=y_{2}(b)=\cdots=y_{n}(b),
$$

where $y_{1}(x)=r(x) y^{(n)}(x)$ as before and

$$
y_{i}(x)=\left(r_{n}(x) y^{(n)}\right)^{(i-1)}+\sum_{j=1}^{i-1}(-1)^{j+1}\left(r_{n-j}(x) y^{(n-j)}\right)^{(i-j-1)}, \quad i=2,3, \cdots, n
$$

Reid's results are stated in the notation of the present discussion.

THEOREM 6.1. (Reid [12]) A necessary and sufficient condition for the nonexistence of $\eta_{1}(0)$ on $(0, c]$ for (1) is that

$$
I_{1}[y ; 0, b]=\int_{0}^{b}\left[r(x)\left(y^{(n)}\right)^{2}-p(x) y^{2}\right] d x>0
$$

for all values of $b$ on $(0, c]$ and all functions $y(x)$ such that $y(x) \in C^{n-1}[0, b]$, $y(x)$ is absolutely continuous on $[0, b],\left(y^{(n)}\right)^{2}$ is integrable on $[0, b]$, and $y(x)$ has $n$ th-order zeros at $x=0$ and $x=b$. Also, if $\eta_{1}(0)$ exists for a solution $y(x)$ of $(1)$, then $I_{1}\left[y ; 0, \eta_{1}(0)\right]=0$.

For the equation (4), the statement analogous to (21) is

$$
I_{2}[y ; 0, b]=\int_{0}^{b}\left[r(x)\left(y^{(n)}\right)^{2}-\sum_{i=0}^{n-1} r_{i}(x)\left(y^{(i)}\right)^{2}\right] d x>0,
$$

where $y(x)$ is the same as in the statement of Theorem 6.1.

TheOREM 6.2. (Reid [12]) A necessary and sufficient condition for the nonexistence of $\mu_{1}(0)$ on $(0, c]$ for (1) is that (21) (or (22) if (4) is used) hold for all values of $b$ on $(0, c]$ and all functions $y(x)$ such that $y(x) \in C^{n-1}[0, b], y(x)$ is absolutely continuous on $[0, b],\left(y^{(n)}\right)^{2}$ is integrable on $[0, b]$ and $y(x)$ has an nth-order zero at $x=0$. Also, if $\mu_{1}(0)$ exists for a solution $y(x)$ of $(1)$, then $I_{1}\left[y ; 0, \mu_{1}(0)\right]=0$.

Theorem 6.1 can now be utilized to obtain the following results.

THEOREM 6.3. If $\eta_{1}(0)$ exists for (1) at $x=x_{1}, r(x) \geqq r_{n}(x)$, and $p(x) \leqq r_{0}(x)$, then $\eta_{1}(0)$ exists for (4) at $x=x_{2}$, say, and $0<x_{2} \leqq x_{1}$.

THEOREM 6.4. If $\mu_{1}(0)$ exists for (1) at $x=x_{1}, r(x) \geqq r_{n}(x)$, and $p(x) \leqq r_{0}(x)$, then $\mu_{1}(0)$ exists for (4) at $x=x_{2}$, say, and $0<x_{2} \leqq x_{1}$.

Now, take $r(x) \equiv r_{n}(x)$ and $p(x) \equiv r_{0}(x)$ on $[0, \infty)$; and then the following results are obtained by combining the results of $\S 4$ and 5 and 
those of the present section.

THEOREM 6.5. If $\int^{\infty} r_{0}(x)\left(\stackrel{x}{I}_{x_{0}}^{n} r_{0}\right)^{2} d x=\infty$, then $\mu_{1}(b)$ exists for (4) for each $b \in[0, \infty)$.

THEOREM 6.6. If $\mu_{1}(0)$ exists for (4) and $\int^{\infty} d x / r_{n}(x)=\infty$, then $\eta_{1}(0)$ exists for (4).

COROLlaRY 6.1. If $\int^{\infty} r_{0}(x)\left(\stackrel{x}{I} r_{0}\right)^{2} d x=\infty$ and $\int^{\infty} d x / r_{n}(x)=\infty$, then, $\eta_{1}(0)$ exists for (4).

Theorem 6.5 and Corollary 6.1 give stronger results than the corresponding results of Reid's [12] since the latter have the condition $\int^{\infty} x^{2(n-1)} r_{0}(x) d x=\infty$ while the former have the weaker condition $\int^{\infty} r_{0}(x)\left({\underset{x}{x}}_{x_{0}}^{I} r_{0}\right)^{2} d x$. (See the proof of Theorem 4.3 for a comparison of these conditions.) Theorem 6.6 can be obtained from Reid's work [12].

\section{BIBLIOGRAPHY}

1. J. H. Barrett, A Prüfer transformation for matrix differential equations, Proc. Amer. Math. Soc., 8 (1957), 510-518.

2. Disconjugacy of a self-adjoint differential equation of the fourth order, Pacific J. Math, 11 (1961), 25-37.

3. - Systems-disconjugacy of a fourth-order differential equation, Technical Summary Report No. 135, Math. Research Center, U. S. Army (1960) 12 pp. Proc. Amer. Math. Soc., 12 (1961), 205-213.

4. - Two-point boundary value problems and comparison theorems for fourth-order self-adjoint differential equations and second-order matrix differential systems, Canadian J. of Math., 13 (1961), 625-638.

5. E. Bodewig, Matrix Calculus, North-Holland Publishing Co., Amsterdam, 1956.

6. E. A. Coddington and N. Levinson, Theory of Ordinary Differential Equations, McGraw Hill, New York, 1955.

7. R. Courant and D. Hilbert, Methods of Mathematical Physics, Vol. I, Interscience, New York, 1953.

8. H. C. Howard, Oscillation criteria for fourth-order linear differential equations, Trans, Amer. Math. Soc., 96 (1960), 296-311.

9. H. Kaufman and R. L. Sternberg, A two-point boundary problem for ordinary selfadjoint differential equations of even order, Duke Math J., 20 (1953), 527-531.

10. Walter Leighton and Z. Nehari, On the oscillation of solutions of self-adjoint linear differential equations of the fourth order, Trans. Amer. Math. Soc., 89 (1958), 325-377.

11. W. T. Reid, A matrix differential equation of the Riccati type, Amer. J. of Math., 68 (1946), 237-246.

12. - Oscillation criteria for self-adjoint differential systems, Trans. Amer. Math. Soc., 101 (1961), 91-106.

13. H. M. Sternberg and R. L. Sternberg, A two-point boundary problem for ordinary selfadjoint differential equations of fourth order, Canadian J. Math., 6 (1954), 416-419.

14. R. L. Sternberg, Variational methods and non-oscillation theorems for systems of differential equations, Duke Math. J., 19 (1952), 311-322. 



\title{
PACIFIC JOURNAL OF MATHEMATICS
}

\author{
EDITORS
}

RalPh S. Phillips

Stanford University

Stanford, California

M. G. Arsove

University of Washington

Seattle 5 , Washington
A. L. Whiteman

University of Southern California Los Angeles 7, California

LOWell J. PAIGE

Unıversity of California

Los Angeles 24, California

\section{ASSOCIATE EDITORS}
E. F. BECKENBACH
D. DERRY
H. L. ROYDEN
E. G. STRAUS
T. M. CHERRY
M. OHTSUKA
E. SPANIER
F. WOLF

\section{SUPPORTING INSTITUTIONS}

\author{
UNIVERSITY OF BRITISH COLUMBIA \\ CALIFORNIA INSTITUTE OF TECHNOLOGY \\ UNIVERSITY OF CALIFORNIA \\ MONTANA STATE UNIVERSITY \\ UNIVERSITY OF NEVADA \\ NEW MEXICO STATE UNIVERSITY \\ OREGON STATE UNIVERSITY \\ UNIVERSITY OF OREGON \\ OSAKA UNIVERSITY \\ UNIVERSITY OF SOUTHERN CALIFORNIA
}

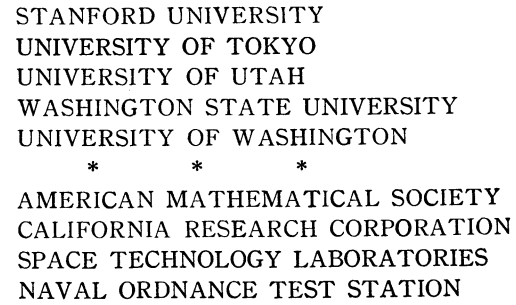

Mathematical papers intended for publication in the Pacific Journal of Mathematıcs should be typewritten (double spaced), and the author should keep a complete copy. Manuscripts may be sent to any one of the four editors. All other communications to the editors should be addressed to the managing editor, L. J. Paige at the University of California, Los Angeles 24, California.

50 reprints per author of each article are furnıshed free of charge; additional copies may be obtained at cost in multiples of 50 .

The Pacific Journal of Mathematics is published quarterly, in March, June, September, and December. Effective with Volume 13 the price per volume (4 numbers) is $\$ 18.00$; single issues, $\$ 5.00$. Special price for current issues to individual faculty members of supporting institutions and to individual members of the American Mathematical Society: $\$ 8.00$ per volume; single issues $\$ 2.50$. Back numbers are available.

Subscriptions, orders for back numbers, and changes of address should be sent to Pacific Journal of Mathematics, 103 Highland Boulevard, Berkeley 8, California.

Printed at Kokusai Bunken Insatsusha (International Academic Printing Co., Ltd.), No. 6 , 2-chome, Fujimi-cho, Chiyoda-ku, Tokyo, Japan.

PUBLISHED BY PACIFIC JOURNAL OF MATHEMATICS, A NON-PROFIT CORPORATION

The Supporting Institutions listed above contribute to the cost of publication of this Journal, but they are not owners or publishers and have no responsibility for its content or policies. 


\section{Pacific Journal of Mathematics}

\section{Vol. 12, No. $3 \quad$ March, 1962}

Alfred Aeppli, Some exact sequences in cohomology theory for Kähler

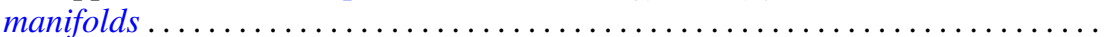

Paul Richard Beesack, On the Green's function of an N-point boundary value

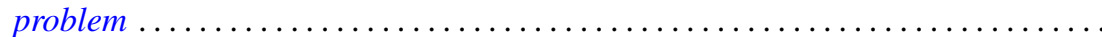

James Robert Boen, On p-automorphic p-groups....

James Robert Boen, Oscar S. Rothaus and John Griggs Thompson, Further results

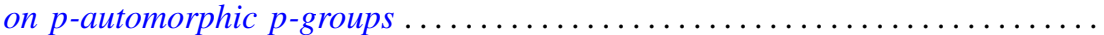

James Henry Bramble and Lawrence Edward Payne, Bounds in the Neumann problem for second order uniformly elliptic operators ..................

Chen Chung Chang and H. Jerome (Howard) Keisler, Applications of ultraproducts of pairs of cardinals to the theory of models .........................

Stephen Urban Chase, On direct sums and products of modules ................

Paul Civin, Annihilators in the second conjugate algebra of a group algebra .......

J. H. Curtiss, Polynomial interpolation in points equidistributed on the unit

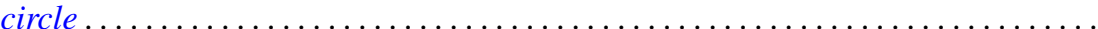

Marion K. Fort, Jr., Homogeneity of infinite products of manifolds with

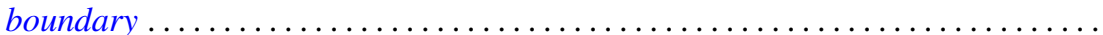

James G. Glimm, Families of induced representations . . . . . . . . . . . . . . .

Daniel E. Gorenstein, Reuben Sandler and William H. Mills, On almost-commuting

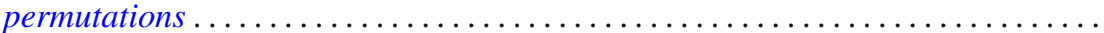

Vincent C. Harris and M. V. Subba Rao, Congruence properties of $\sigma_{r}(N) \ldots \ldots \ldots$

Harry Hochstadt, Fourier series with linearly dependent coefficients . . . . . . . . . . 925

Kenneth Myron Hoffman and John Wermer, A characterization of $C(X)$. .

Robert Weldon Hunt, The behavior of solutions of ordinary, self-adjoint differential equations of arbitrary even order...

Edward Takashi Kobayashi, A remark on the Nijenhuis tensor

David London, On the zeros of the solutions of $w^{\prime \prime}(z)+p(z) w(z)=0$

Gerald R. Mac Lane and Frank Beall Ryan, On the radial limits of Blaschke products...

T. M. MacRobert, Evaluation of an E-function when three of its upper parameters

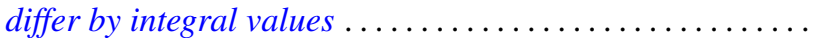

Robert W. McKelvey, The spectra of minimal self-adjoint extensions of a symmetric operator

Adegoke Olubummo, Operators of finite rank in a reflexive Banach space. .

David Alexander Pope, On the approximation of function spaces in the calculus of variations

Bernard W. Roos and Ward C. Sangren, Three spectral theorems for a pair of singular first-order differential equations...............

Arthur Argyle Sagle, Simple Malcev algebras over fields of characteristic zero .

S. C. Tang, Some theorems on the ratio of empirical distribution to the theoretical distribution

Robert Charles Thompson, Normal matrices and the normal basis in abelian number fields.

Howard Gregory Tucker, Absolute continuity of infinitely divisible distributions ... 